\title{
Gestão da Atenção Básica em Saúde Bucal no Município de Fortaleza, Ceará, entre 1999 e 2006
}

\section{Primary Health Care Management in Oral Health in Fortaleza, Ceará, between 1999 and 2006}

\author{
Caroline Ferreira Martins Lessa \\ Mestre em Vigilância em Saúde. Cirurgiã-dentista da Secretaria \\ Municipal de Saúde de Fortaleza-Ceará. \\ Endereço: Rua Egidio de Oliveira, 950, Bairro José de Alencar, CEP \\ 60833-840, Fortaleza, CE, Brasil. \\ E-mail: carolfm.lessa®gmail.com \\ Mario Vianna Vettore \\ Doutor em Saúde Pública. Professor Adjunto do Instituto de Estudos \\ em Saúde Coletiva Universidade Federal do Rio de Janeiro \\ Endereço: IESC/UFRJ. Praça Jorge Machado Moreira, Ilha do Fundão, \\ Cidade Universitária, CEP 21944-970, Rio de Janeiro, RJ, Brasil. \\ E-mail: marioळensp.fiocruz.br
}

\section{Resumo}

Este estudo analisou o grau de adequação da gestão da Atenção Básica $(\mathrm{AB})$ em saúde bucal no município de Fortaleza, Ceará, entre 1999 e 2006. A metodologia empregada teve como base uma matriz de análise de cinco dimensões: Planejamento e Programação, Suporte da Gestão aos Profissionais e às Práticas na $\mathrm{AB}$, Oferta de Assistência Odontológica, Utilização de Serviços e Integralidade da Atenção. As informações foram obtidas de planos municipais de saúde, de relatórios de gestão e análise de dados secundários do Sistema de Informação Ambulatorial (SIA-SUS) e do IBGE. De maneira complementar, a partir da análise dos indicadores do Pacto da $\mathrm{AB}$ (2006), obtiveram-se informações com gestores de saúde bucal. Observou-se uma melhora nas quatro primeiras dimensões da matriz de análise, e apenas a dimensão relativa à utilização de serviços declinou no período. A tendência dos indicadores de saúde bucal foi heterogênea. Enquanto a "Cobertura de Primeira Consulta odontológica” reduziu, a "Proporção de procedimentos odontológicos especializados em relação às ações individuais da $A B$ ” apresentou discreto aumento. Concluiu-se que o município de Fortaleza apresentou adequação da gestão da $\mathrm{AB}$ em saúde bucal ao modelo de vigilância em saúde, entre 1999 e 2006. Entretanto, o declínio na utilização de serviços odontológicos associado à estabilidade dos indicadores de cobertura e assistência odontológica sugere a necessidade de sistematizar atividades de planejamento e avaliação das ações de saúde bucal na $\mathrm{AB}$ em Fortaleza.

Palavras-chave: Saúde bucal; Sistema Único de Saúde, Atenção primária à saúde; Gestão em saúde; Indicadores básicos de saúde. 


\section{Abstract}

This study assessed the degree of adequacy of Primary Care management in oral health in the city of Fortaleza, state of Ceará, Brazil, between 1999 and 2006. The employed methodology was based on a matrix of analysis including five dimensions: Planning and Programming, Management Support to Primary Care Professionals and Practices, Dental Assistance Offered, Health Services Utilization and Integrality of Care. The information was obtained from municipal health plans, management reports and analysis of secondary data from Sistema de Informação Ambulatorial (SIA-SUS - Outpatient Clinic Information System of Brazil's National Health System) and from IBGE (Brazilian Institute of Geography and Statistics). In addition, health indicators from the Primary Health Pact (2006) and information from oral health managers were analyzed. An improvement was observed in the first four dimensions of the matrix of analysis and only the dimension related to health services utilization declined during the period of the study. The trend of the oral health indicators was heterogeneous. While the oral health indicator "Coverage of the First Dental Consultation" decreased, the "Proportion of Specialized Dental Procedures in Relation to Individual Actions in Primary Care" showed a slight increase. It was concluded that the Primary Care management in oral health in the city of Fortaleza became adequate to the health surveillance model between 1999 and 2006. However, the decline in dental health services utilization, associated with the stability of the indicators of dental coverage and assistance, suggest the need to systematize the activities of planning and assessment of oral health actions in Primary Care in Fortaleza.

Keywords: Oral Health; National Health System; Primary Health Care; Health Management; Basic Health Indicators.

\section{Introdução}

A Política Nacional de Saúde Bucal do Ministério da Saúde, implementada em 2004, reorientou o modelo de atenção em saúde bucal, reforçando a necessidade de integração da odontologia no contexto da Atenção Básica. O planejamento, a avaliação e o monitoramento das ações, bem como o uso de indicadores de saúde bucal no Pacto de Indicadores da Atenção Básica passaram a ser ferramentas importantes para a organização da saúde bucal na Atenção Básica (Brasil, 2006a).

Em 2006, foram propostos indicadores em saúde bucal importantes para o monitoramento das ações nessa área, e o seu emprego e metas a serem alcançadas foram pactuados por estados e municípios (Brasil, 2006a). Em 2007, a Portaria Ministerial $n^{\circ}$. 91/GM estabeleceu o Pacto pela Saúde, que unificou o Pacto pela Atenção Básica e aqueles dos demais níveis de complexidade (Brasil, 2007a).

Os principais sistemas que registram dados da Atenção Básica em saúde bucal são o Sistema de Informação da Atenção Básica (SIAB) e o Sistema de Informação Ambulatorial do Sistema Único de Saúde (SIA-SUS), ambos do Ministério da Saúde. Em alguns municípios, o SIA-SUS é o único sistema que, embora tenha surgido para fins de pagamento, oferece os dados referentes aos procedimentos ambulatoriais (Scatena e Tanaka, 2001).

O modelo de atenção em saúde bucal deve considerar as características municipais. A organização do planejamento e da programação dos serviços de saúde pressupõe o emprego de instrumentos normativos, incluindo Planos Municipais de Saúde constando metas e meios para alcançá-las. Ademais, a efetividade da atenção está relacionada com a gestão ou política pública baseada em evidências e com o estabelecimento de contratos negociados entre gestores e profissionais que, por sua vez, são capazes de influenciar os indicadores de saúde (Brasil, 2007b).

A avaliação e o monitoramento das ações em saúde bucal são ferramentas relevantes para a organização das ações de saúde bucal na Atenção Básica. Assim, considerando os conceitos atuais na organização da atenção em saúde bucal, o presente trabalho analisou o grau de adequação da gestão da Atenção Básica em saúde bucal, considerando o 
modelo de reorientação da atenção em saúde bucal no município de Fortaleza, Ceará, de 1999 a 2006.

\section{Método}

\section{Desenho do estudo e seleção do caso}

Realizou-se um estudo de caso instrumental no qual foi avaliada a adequação da gestão da Atenção Básica em saúde bucal ao modelo de reorientação da atenção em saúde bucal no município de Fortaleza, no período de 1999 a 2006 (Alves-Mazzotti, 2006). O município de Fortaleza caracteriza-se pela adesão tardia à Estratégia de Saúde da Família (ESF), além da incorporação de outras propostas de modelo de atenção para a estruturação do nível primário entre 1999 e 2004, e o incentivo à ESF a partir de 2005.

Foram definidos quatro momentos comparativos da gestão da Atenção Básica (AB) em saúde bucal no município: 1999-2000, 2001-2002, 2003-2004 e 2005-2006. A agregação em biênios baseou-se na realidade local, caracterizada por distintos programas implantados e políticas públicas vigentes em diferentes momentos (fonte: http://www.sms. fortaleza.ce.gov.br/sms_v2/).

\section{Modelo teórico-lógico}

Para avaliar a adequação da gestão da $\mathrm{AB}$ em saúde bucal ao modelo de reorientação da atenção em saúde bucal foram adotadas três estratégias: matriz de análise, avaliação dos gestores em saúde bucal e análise de indicadores de saúde bucal na AB.

Para avaliar a gestão da $\mathrm{AB}$ em saúde bucal empregou-se a matriz de análise adaptada com as dimensões Planejamento e programação, Suporte da gestão aos profissionais e às práticas na $\mathrm{AB}$, Oferta de assistência odontológica, Utilização de serviços e Integralidade da atenção (Chaves e Vieira-da-Silva, 2007).

De forma complementar, foram investigados os gestores de saúde bucal. Além disso, foram construídos os indicadores de saúde bucal do Pacto de Indicadores da $\mathrm{AB} 2006$ para o período do estudo (Brasil, 2006b).

\section{Método de coleta e fonte de dados: aplicação da matriz de análise}

Para o emprego da matriz de análise, realizou-se inicialmente um estudo documental de instrumentos normativos de gestão da Secretaria Municipal de Saúde (SMS) e do Conselho Municipal de Saúde de Fortaleza, Ceará. Foram também coletados documentos empregados diretamente na gestão em saúde bucal com o objetivo de identificar quais eram esses instrumentos, além de complementar as informações dos documentos principais. Os instrumentos de gestão coletados foram os relatórios de gestão e os planos municipais de saúde, identificados para os biênios 2001-2002 e 2003-2004. Para o primeiro biênio, 1999-2000, não foram encontrados os relatórios de gestão, sendo utilizados outros documentos, como planos de ação e relatórios. Em 2005-2006, apenas os relatórios de gestão foram empregados, uma vez que o plano municipal de saúde que envolve 2006 não havia sido concluído até o final da coleta dos dados, e o ano de 2005 não foi mencionado no plano anterior.

As informações obtidas nesses documentos foram utilizadas integralmente nas dimensões Planejamento e Programação, Suporte da gestão aos profissionais e às práticas na $\mathrm{AB}$, e parcialmente nas dimensões Oferta de assistência odontológica e Integralidade da atenção.

Para a dimensão "Oferta da assistência odontológica” foram agregados dados coletados nos documentos de gestão (número de dentistas no setor público) e do IBGE (população com renda per capita inferior a 50\% do salário mínimo). Em relação à "Integralidade da Atenção", utilizaram-se dados dos documentos de gestão (existência de sistema de referência e serviços ofertados) e dados do SIA-SUS (procedimentos especializados em odontologia).

Além disso, dados secundários do SIA-SUS sobre os procedimentos de saúde bucal foram utilizados para responder à dimensão "Utilização de Serviços".

\section{Caracterização do nível de adequação da gestão da Atenção Básica em saúde bucal}

A caracterização da gestão em níveis de adequação foi baseada exclusivamente nos resultados das dimensões da matriz de análise. As dimensões abordaram o planejamento com enfoque epidemiológico e a avaliação das ações, o suporte da gestão aos profissionais e às práticas na Atenção Básica, a utilização de serviços e a integralidade. Para cada uma das dimensões foi atribuído um escore que 
permitiu caracterizar o grau de adequação da gestão em saúde bucal no município de Fortaleza (Chaves e Vieira-da-Silva, 2007).

A análise foi realizada para cada um dos quatro momentos preestabelecidos e cada período foi classificado em três níveis de adequação: incipiente (menor que 33,3\% da pontuação máxima), intermediário (superior ou igual a 33,3\% e inferior ou igual a $66,6 \%$ da pontuação máxima) e avançado (superior a $66,6 \%$ da pontuação máxima proposta).

\section{Informações dos gestores em saúde bucal}

As informações dos gestores em saúde bucal foram obtidas através de questionários autopreenchidos e semiestruturados. As mesmas informações contidas nos parâmetros das dimensões da matriz de análise foram coletadas, como também o perfil dos gestores. Três gestores foram identificados através de informantes-chave da própria SMS de Fortaleza.

O questionário foi pré-testado em dois gestores municipais em saúde bucal, sendo um do estado do Ceará, efetuando-se as adaptações necessárias. 0 questionário foi testado novamente em outros nove gestores municipais em saúde bucal do estado do Ceará, não sendo necessária nenhuma modificação. Essas informações foram analisadas de forma complementar para uma maior compreensão das dimensões propostas.

\section{Construção dos indicadores de saúde bucal}

Para a construção dos indicadores do Pacto de Indicadores da $\mathrm{AB}$ 2006, empregaram-se os dados do SIA-SUS para os anos de 1999 a 2006 (Brasil, 2006b). A escolha desses instrumentos foi baseada nas diretrizes do Ministério da Saúde, que instituiu esses mecanismos como aqueles de escolha para o acompanhamento das ações de saúde bucal no país (Brasil, 2006a).

Embora esse pacto tenha sido estabelecido em 2006, os indicadores propostos foram calculados para todos os anos do período do estudo. Os indicadores utilizados foram: Cobertura de Primeira Consulta Odontológica, Média de procedimentos odontológicos básicos individuais e Proporção de procedimentos odontológicos especializados em relação às ações individuais da $\mathrm{AB}$ em saúde bucal (Brasil, 2006b).

Para o cálculo de todos os indicadores de saúde bucal, o total populacional foi obtido do Censo Demográfico 2000 do IBGE (www.ibge.org.br). A população de interesse pode ser considerada estável, o que minimizou a ocorrência de vieses nas estimativas dos indicadores.

O cálculo dos indicadores de saúde bucal para o período compreendido entre janeiro de 1999 e outubro de 1999, período em que os códigos do SIA-SUS não correspondiam aos atuais, foi feito com base em códigos do SIA-SUS equivalentes.

\section{Aspectos éticos da pesquisa}

O estudo foi aprovado pelo Comitê de Ética e Pesquisa da Escola Nacional de Saúde Pública Sergio Arouca, Fundação Osvaldo Cruz (Parecer n ${ }^{0} 180 /$ o 8 , de 10/02/2008).

\section{Resultados}

Caracterização do nível de adequação da gestão da Atenção Básica em saúde bucal segundo a pontuação das dimensões da matriz de análise

Os relatórios de gestão em saúde foram encontrados para todos os anos em estudo, com exceção de 1999 e 2000. Quanto aos planos municipais, estiveram acessíveis aqueles que envolveram os três primeiros biênios, 1999-2000, 2001-2002 e 2003-2004. Ressalta-se que o plano municipal de saúde para o período entre 2006 e 2009 não foi encontrado, pois não foi concluído até o momento da coleta de dados. 0 ano de 2005 não foi mencionado no plano municipal anterior. 0 detalhamento dos documentos levantados na coleta de dados está expresso no Quadro 1.

A classificação do grau de adequação da gestão da Atenção Básica em saúde bucal nos diferentes períodos é apresentada na Tabela 1. No período de 1999 a 200o, as dimensões "Planejamento e Programação", "Suporte da gestão aos profissionais e às práticas na Atenção Básica” e "A Integralidade da atenção" apresentaram adequação incipiente. Desempenho um pouco melhor (intermediário) foi encontrado nas dimensões relativas a "A oferta da assistência odontológica (cobertura potencial)" e "Utilização de Serviços (cobertura real)". Entre 2001 e 2002, percebeu-se um avanço nas dimensões "Suporte da gestão aos profissionais e às práticas na Atenção Básica", "A oferta da assistência odontológica (co- 


\section{Quadro I - Instrumentos normativos empregados pela gestão na Atenção Básica em saúde bucal no município de Fortaleza, Ceará, entre 1999 e 2006}

\begin{tabular}{|c|c|c|c|c|}
\hline & $1999-2000$ & $2001-2002$ & $2003-2004$ & $2005-2006$ \\
\hline Gestão da Saúde & $\begin{array}{l}\text { - Plano de Ação } 2000 \\
\text { - Ações estratégicas } \\
\text { intersetoriais } \\
\text { Indicadores de saúde } \\
\text { atingidos em } 1999 \text { e } \\
2000 \text { com meta para } \\
200 \text { I } \\
\text { Relatório do PSF - } \\
\text { Fortaleza, Jun. } 2000 . \\
\text { - Plano Municipal de } \\
\text { Saúde 1998-200। }\end{array}$ & $\begin{array}{l}\text { - Relatório de Gestão } \\
2001 \\
\text { - Relatório de Gestão } \\
2002 \\
\text { - Plano Municipal de } \\
\text { Saúde } 2001-2004\end{array}$ & $\begin{array}{l}\text { - Relatório de Gestão } 2003 \\
\text { - Relatório de Gestão } 2004 \\
\text { - Plano Municipal de Saúde } \\
2001-2004\end{array}$ & $\begin{array}{l}\text { - Relatório de Gestão } \\
2005 \\
\text { - Relatório de Gestão } \\
2006\end{array}$ \\
\hline Gestão da Saúde Bucal & $\begin{array}{l}\text { - Instrumento de } \\
\text { Acompanhamento das } \\
\text { ações preventivas/ } \\
\text { educativas nas escolas } \\
\text { patrimoniais de } \\
\text { Fortaleza } 1999 \text { e } 2000\end{array}$ & $\begin{array}{l}\text { - Consolidado de } \\
\text { Procedimentos } \\
\text { odontológicos } 2001 \\
\text { - Consolidado Mensal } \\
\text { de Procedimentos } \\
\text { odontológicos } 2002\end{array}$ & $\begin{array}{l}\text { - Planilha de Acompanhamento } \\
\text { Mensal de Projetos } 2003 \\
\text { - Avaliação do BPA Mensal } \\
2004 \\
\text { - Planilha de Acompanhamento } \\
\text { Mensal da Capacidade } \\
\text { Instalada e Procedimentos } \\
\text { realizados com base na meta } \\
\text { mensal programada e \% } \\
\text { atingido } 2003 \text { e } 2004\end{array}$ & $\begin{array}{l}\text { - Avaliação do BPA } \\
\text { Mensal } 2005 \text { e } 2006 \\
\text { - Avaliação das } \\
\text { Ações coletivas } 2005 \\
\text { e } 2006 \text { (produção e } \\
\text { cobertura) }\end{array}$ \\
\hline
\end{tabular}

bertura potencial)" e "A Integralidade da atenção" que passaram dos níveis incipiente, intermediário e incipiente para avançado, avançado e intermediário, respectivamente. 0 nível de adequação desse período foi mantido no biênio 2003-2004. No último momento do estudo, de 2005 a 2006, identificou-se o primeiro avanço na dimensão "Planejamento e Programação", que obteve nos momentos anteriores o mesmo nível de adequação (incipiente), atingindo o nível intermediário nesse biênio. Outro aspecto positivo identificado corresponde a "A Integralidade da atenção", que nos anos de 2003-2004 obteve nível intermediário de adequação, passando para o nível avançado de adequação. Entretanto, identificou-se queda nos resultados referentes à "Utilização de Serviços (cobertura real)", caindo do nível intermediário obtido nos três momentos anteriores para o nível incipiente de adequação.

No entanto, o "peso" de cada dimensão nos biênios estudados pode ser visualizado na Figura 1, em que se percebe a evolução da dimensão "Planejamento e Programação" no último biênio quando comparado aos demais períodos. Nota-se também o aumento gradual da cobertura potencial, embora os valores absolutos finais obtidos para 2001-2002 e 2003-2004 reflitam uma queda $(3,5 \times 5,0)$, o que não ficou evidente através da comparação do nível de adequação, pois o ponto de corte utilizado classifica a pontuação 3,5 como representando nível avançado. A cobertura real apresentou uma queda expressiva nos últimos anos. Quanto à "Integralidade da atenção", observou-se um aumento do primeiro para o segundo biênio, uma regularidade entre os dois biênios e um aumento considerável nos últimos anos.

\section{Análise das informações dos gestores de saúde bucal}

Foram identificados três responsáveis, ou gestores, pelo setor saúde bucal no período de estudo: um de 2002 (início não informado) a agosto de 2003; outro entre setembro de 2003 e dezembro de 2004 , e o terceiro de janeiro de 2005 ao final de 2006. A idade dos gestores variou entre 36 e 51 anos, e dois eram do sexo feminino. Em relação à escolaridade, todos apresentavam curso superior de odontologia e especialização em saúde pública ou áreas correlatas. 
Figura I - Comparação da representação das dimensões analisadas segundo os momentos do período do estudo, 1999-2006, Fortaleza, Ceará

Escore do grau de adequação da gestão em saúde bucal de acordo com Chaves e Vieira-da-Silva , 2007.

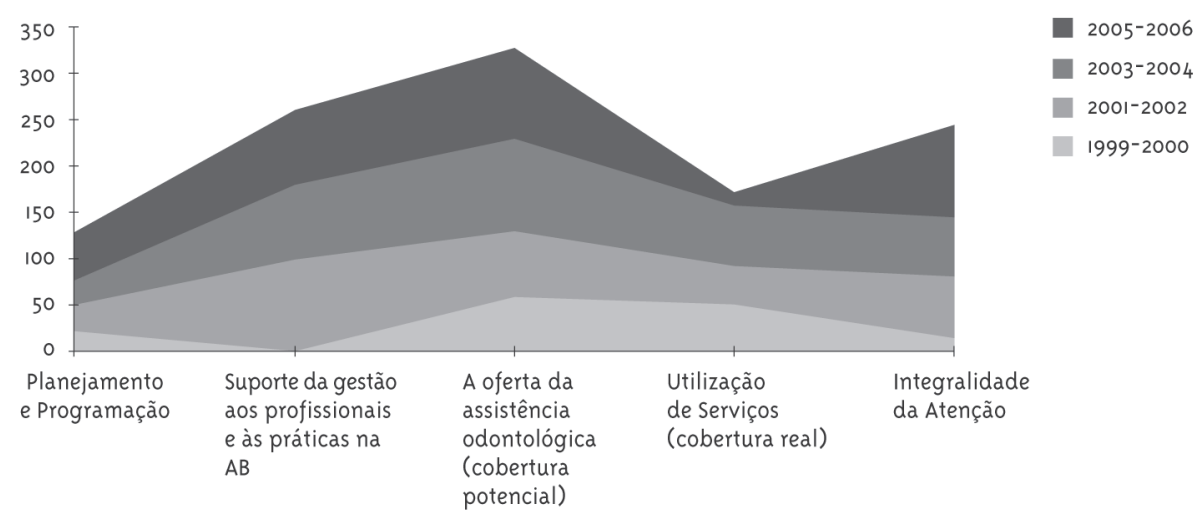

As dimensões exploradas através do questionário aplicado aos gestores ou responsáveis pela saúde bucal foram "Planejamento e Programação", "O suporte da gestão aos profissionais e às práticas na atenção básica" e a "Integralidade da atenção".

No item referente à realização de levantamento epidemiológico, parte integrante da análise da dimensão "Planejamento e Programação", os gestores dos dois últimos biênios afirmaram ter ocorrido esta atividade durante a sua gestão, apontando como mecanismos para a divulgação e a incorporação dos resultados pelas equipes da Atenção Básica os informativos, as reuniões, as oficinas regionais, a criação de núcleos para a divulgação dos dados, os relatórios e as oficinas. 0 planejamento das ações em saúde bucal bem como a avaliação dessas atividades, quesitos também considerados na dimensão "Planejamento e Programação", foram ações apontadas por gestores nos períodos 2003-2004 e 2005-2006.

A realização da supervisão do trabalho desenvolvido pelas equipes e o suporte material e instrumental para as atividades e ações da $A B$ em saúde bucal, itens da dimensão "O suporte da gestão aos profissionais e às práticas na atenção básica”, estiveram presentes entre 2003 e 2006.

Em relação aos sistemas de referência para outros níveis de complexidade e serviços disponíveis, todos informaram a existência de um sistema de referência das unidades básicas para a média complexidade. Os serviços disponíveis mais frequentes foram: endodontia, complementação diagnóstica com $\mathrm{Rx}$, prótese total e prótese parcial removível. Estomatologia/diagnóstico do câncer foram encontradas em dois questionários. No último biênio foi especificado haver referência para as áreas de prótese unitária, prótese fixa, periodontia e odontopediatria.

Em relação aos recursos humanos disponíveis para a gestão da saúde bucal, apenas o gestor do período 2005-2006 informou que contava com uma equipe de assessores nas áreas de vigilância em saúde, além de coordenadores regionais. Os demais assinalaram que não havia responsável formal e que o assessor técnico em saúde bucal estava subordinado à coordenação da Atenção Básica.

Quanto às formas de organização da Atenção Básica, todos os gestores informaram a existência da ESF e do programa de agentes comunitários de saúde, além de unidades de saúde com programas específicos para saúde da mulher e da criança, controle da diabetes e da hipertensão. Foi registrado ainda haver unidades de saúde sem programas de saúde pública implantados entre 2002 e 2004, e o pronto-atendimento em 2005-2006.

Outra informação adicional obtida corresponde ao repasse e à orientação dada às equipes de saúde bucal na $\mathrm{AB}$ sobre a alimentação e o uso dos sistemas de informação SIA-SUS e SIAB. Os gestores de 2003-2004 e 2005-2006 afirmaram terem realizado esta atividade. 


\section{Indicadores de Saúde Bucal da Atenção Básica}

O indicador "Cobertura de Primeira Consulta Odontológica” apresentou declínio em 2000 e 2001, tendo como linha de base o ano de 1999. A partir de 2001, verificou-se aumento de quase duas vezes no valor do indicador, voltando a decrescer nos anos seguintes até o final do estudo (2002-2006). Quanto à "Média de procedimentos odontológicos básicos individuais" observou-se tendência de aumento a partir de 1999 até 2002. Após este ano, entre 2003 e 2005 , verificou-se redução nesse indicador, e, em 2006, percebeu-se aumento de aproximadamente 50\% em seu valor em relação a 2005 (Figuras 2 e 3).

Figura 2 - Comportamento temporal dos indicadores "Cobertura de Primeira Consulta Odontológica" ( 1 ) e "Proporção de procedimentos odontológicos especializados em relação às ações individuais da Atenção Básica" (2) no município de Fortaleza, Ceará, 1999-2006

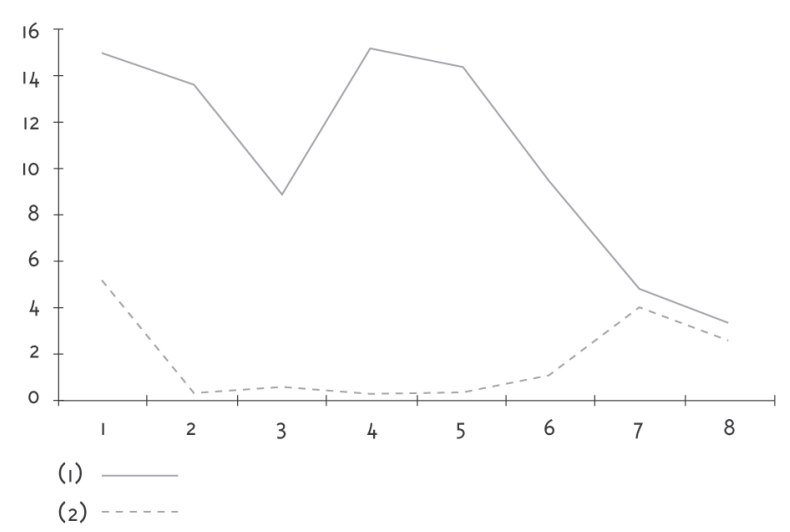

Figura 3 - Comportamento temporal do indicador "Média de procedimentos odontológicos básicos individuais" no município de Fortaleza, Ceará, 1999-2006

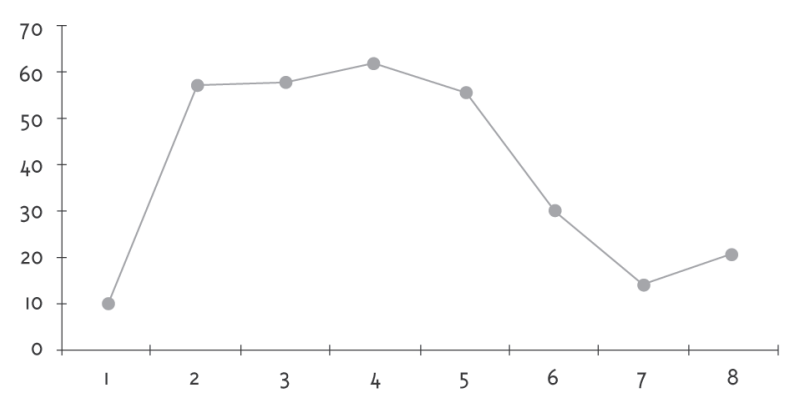

Em relação ao indicador "Proporção de procedimentos odontológicos especializados em relação às ações individuais da Atenção Básica”, percebeu-se uma redução comparando 1999 e 200o, seguindo com uma variação nos valores finais anuais entre 2001 e 2004. Em 2005, observou-se aumento de $100 \%$ se considerado o resultado de 2004 , e em 2006, valor muito próximo ao ano de 2005. As tendências de mudanças dos indicadores estão apresentadas nas Figuras 2 e 3.

\section{Discussão}

Apesar de a avaliação das políticas e programas estar presente na agenda do Ministério da Saúde, observase que são incipientes as iniciativas de avaliação da gestão da Atenção Básica em saúde bucal.

A avaliação da adequação da gestão da Atenção Básica em saúde bucal em Fortaleza, entre 1999 e 2006, através da matriz de análise validada, demonstrou que houve avanços em quatro das cinco dimensões analisadas. Entretanto, a dimensão "Utilização de Serviços (cobertura real)" apresentou tendência de declínio ao longo do estudo, em especial nos anos de 2005 e 2006, apesar dos investimentos na ampliação das equipes de saúde bucal na ESF desde 2005. Situação semelhante foi observada na Bahia, onde um dos municípios analisados, também com aumento na oferta de serviços, não apresentou esse aumento proporcional nos indicadores de produção de serviços ambulatoriais no SIA-SUS (Chaves e Vieira-da-Silva, 2007).

Para a análise dos quesitos da dimensão "Utilização de Serviços (cobertura real)”, os dados do SIA-SUS representaram a principal fonte de informação, ao passo que para as demais dimensões foram empregadas, essencialmente, informações dos documentos de gestão. Embora o SIA-SUS seja um importante sistema de informação para a saúde bucal na Atenção Básica, existe pouca tradição no emprego dessa ferramenta no planejamento e na avaliação das ações odontológicas (Scatena e Tanaka, 2001; Chaves e Vieira-da-Silva, 2007; Barros e Chaves, 2003). A avaliação rotineira das ações implementadas pela gestão em saúde bucal foi uma deficiência observada neste estudo e também foi apontada em um estudo na Bahia (Chaves e Vieira-da-Silva, 2007). 
É possível que a pouca utilização do SIA-SUS no município do estudo possa ter influenciado na mudança negativa no comportamento temporal da dimensão relativa à utilização de serviços. Essa deficiência parece ter influenciado o desempenho do último biênio. No entanto, deve-se ter cautela nessas interpretações, pois a pouca utilização dos Sistemas de Informação em Saúde, especialmente em saúde bucal, pode ter criado uma superestimação dos procedimentos odontológicos nos anos iniciais do estudo ou mesmo uma subestimação dos dados no final (Brasil, 2007c). A disponibilidade de computadores e recursos associados pode ter papel importante no acesso a estes dados. A redução na utilização de serviços possivelmente está relacionada à estrutura física de consultórios odontológicos e materiais de consumo, necessários à manutenção da oferta de serviços odontológicos à população assistida.

Como mencionado, a análise das outras quatro dimensões envolveu fontes de informação variadas, como dados primários de documentos de gestão e dados do IBGE. A partir de 2000, o incentivo à inserção do cirurgião-dentista na Atenção Básica através da ESF gerou novas demandas nas secretarias de saúde, criando a necessidade de equipes de técnicos voltados à gestão dessas ações (Brasil, 200o). Ademais, foram criados incentivos financeiros e foram estabelecidas metas através dos pactos de indicadores. Ao longo desse período, houve o estímulo à realização de levantamentos epidemiológicos nos estados e municípios, como também a criação de centros de referência da atenção especializada em odontologia (Brasil, 2004a; Brasil, 2004b). Todas essas iniciativas devem ter exercido influência positiva sobre as quatro dimensões que apresentaram melhora ao longo do estudo, as quais consideraram o planejamento das ações, o apoio às ações na Atenção Básica, a oferta da assistência e a integralidade da atenção.

Embora a dimensão "Planejamento e Programação" tenha melhorado no último biênio, ainda apresentou nível de adequação intermediário. Este achado foi observado por Padilha e colaboradores (2005) em um estudo na Paraíba, onde ações adequadas de planejamento e programação foram raras.

Foi possível perceber algumas tendências simi- lares entre a análise das dimensões da matriz de análise e a dos indicadores de saúde bucal, como a queda no indicador de "Primeira Consulta Odontológica" e a piora na dimensão "Utilização de serviços", especialmente em 2005 e 2006 . Houve também melhora da "Proporção de procedimentos odontológicos especializados em relação às ações individuais da Atenção Básica" bem como da dimensão "Integralidade da atenção" no último biênio investigado.

Essa associação permite supor que há deficiências na utilização dos serviços odontológicos nesses últimos anos. Fato esse corroborado ainda pela avaliação dos procedimentos coletivos por habitante/ano, quesito da dimensão "Utilização de Serviços", a qual se manteve aquém do estabelecido como padrão mínino: quatro procedimentos coletivos habitante/ano na população geral em todos os biênios estudados.

A melhora observada na dimensão "Integralidade da Atenção", como no indicador que mede a relação entre ações especializadas e Atenção Básica, pode estar refletindo que no cotidiano dos serviços a atenção secundária está recebendo mais incentivo. Entretanto, essa observação exige novos estudos para Fortaleza, pois o maior volume de serviços da atenção especializada não é ofertado pelo município e, sim, pela Secretaria de Saúde do estado do Ceará.

O perfil dos gestores não alterou significativamente ao longo do estudo, entretanto, houve mudança na adequação da gestão e no desempenho dos indicadores.

A avaliação da gestão em saúde bucal tem sido objeto de outras investigações. Um estudo em dois municípios baianos investigou em que medida a descentralização da gestão da atenção influenciou a organização dos serviços odontológicos. A análise da gestão da atenção em saúde bucal e as práticas dos profissionais na $A B$ revelaram que mesmo nos casos em que houve descentralização avançada da gestão, esta não foi acompanhada de melhorias na capacidade de gestão dos serviços odontológicos (Chaves e Vieira-da-Silva, 2007).

Em outro estudo na Bahia, buscou-se identificar a influência de fatores ligados à gestão da atenção à saúde bucal, à formação, à inserção e ao perfil 
dos profissionais sobre a prática profissional. 0 padrão de organização do processo de trabalho foi influenciado pelas características da gestão, na qual municípios com maior articulação entre ações clínicas individuais, coletivas, preventivas e de planejamento apresentaram organização do trabalho mais próxima aos princípios do SUS (Chaves e Silva, 2007).

O estudo de Souza e Roncalli (2007) em municípios do Rio Grande do Norte analisou a incorporação da saúde bucal na ESF. A avaliação das ações de saúde bucal foi apontada como uma medida estritamente administrativa, pouco compartilhada pelos gestores com as equipes de saúde bucal. As medidas de avaliação usualmente empregadas pelos municípios constituíram-se como métodos frágeis, uma vez que há poucos indicadores específicos para a saúde bucal como também registros incorretos nos SIA-SUS pelos municípios.

Embora a avaliação em saúde bucal ainda seja incipiente nos serviços de saúde, a agregação de metodologias, a exploração dos sistemas de informação e a utilização de documentos de gestão podem contribuir no aprimoramento dessa prática.

\section{Referências}

ALVES-MAZZOTTI, A. J. Usos e abusos dos estudos de caso. Cadernos de Pesquisa, São Paulo, v. 36, n. 129, p. 637-651, 2006.

BARROS, S. G.; CHAVES, S. C. L. A utilização do Sistema de Informações Ambulatoriais (SIASUS) como instrumento para caracterização das ações de saúde bucal. Epidemiologia e Serviços de Saúde, Brasília, DF, v. 12, n. 1, p. 41-51, 2003.

BRASIL. Ministério da Saúde. Portaria nº 1444, de 28 de dezembro de 200o. Estabelece incentivo financeiro para a reorganização da atenção á saúde bucal prestada nos municípios por meio do Programa de saúde da Família. Diário Oficial da União, Brasília, DF, 20oo. Disponível em: <http://www.mp.go.gov.br/portalweb/hp/2/docs/ portaria1444_28_12_oo.pdf >. Acesso em: 15 ago. 2007.
BRASIL. Ministério da Saúde. Secretaria de Atenção À Saúde. Departamento de Atenção Básica. Coordenação Nacional de Saúde Bucal. Diretrizes da política nacional de saúde bucal. Brasília, DF, 2004a. Disponível em: <http://bvsms. saude.gov.br/bvs/publicacoes/politica_nacional_ brasil_sorridente.pdf $>$. Acesso em: 27 ago. 2007.

BRASIL. Ministério da Saúde. Secretaria de Atenção à Saúde. Departamento de Atenção Básica. SB Brasil: levantamento das condições de saúde bucal da população brasileira. Informe da Atenção Básica, Brasília, DF, ano V, n. 23, julho/agosto. 2004b. Disponível em: <http://200.214.130.35/dab/docs/publicacoes/ informes/psfinfo23.pdf >. Acesso em: 27 ago. 2007.

BRASIL. Ministério da Saúde. Secretaria de Atenção à Saúde. Departamento de Atenção Básica. Cadernos de Atenção Básica: saúde bucal, Brasília, DF, 20o6a. Disponível em: <http:// dtr2oo4.saude.gov.br/dab/caderno_ab.php>. Acesso em: 16 ago. 2007.

BRASIL. Ministério da Saúde. Portaria n 493 / MS, de 10 de março de 2006. Aprova a relação de indicadores da atenção básica 2006, cujos indicadores deverão ser pactuados entre municípios, estados e o Ministério da Saúde. Diário Oficial da União, Brasília, DF, 20o6b. Disponível em: <dtr2oo4.saude.gov.br/dab/docs/ legislacao/portaria91_10_01_07.pdf >. Acesso em: 26 ago. 2007.

BRASIL. Ministério da Saúde. Portaria nº. 91/ MS, de 10 de janeiro de 2007. Regulamenta a unificação do processo de pactuação de indicadores do Pacto pela Saúde, a serem pactuados por municípios, estados e Distrito Federal. Diário Oficial da União, Brasília, DF, 2007a. Disponível em: <dtr2004.saude.gov.br/dab/ docs/legislacao/portaria91_10_01_07.pdf >. Acesso em: 15 ago. 2007.

BRASIL. Ministério da Saúde. Gestão da Atenção Básica: como organizar um sistema de saúde liderado pela Atenção Básica/ Saúde da Família. Revista Brasileira Saúde Família, Brasília, DF, n. 14, p. 10-17, 2007b. 
BRASIL. Ministério da Saúde. Secretaria de Vigilância em Saúde. Departamento de Análise de Situação de Saúde. Guia metodológico de avaliação e definição de indicadores: doenças crônicas não transmissíveis e rede Carmen. Brasília, DF, 2007c. Disponível em: <http://portal. saude.gov.br/portal/arquivos/pdf/guia_rede carmen.pdf >. Acesso em: 25 ago. 2007.

CHAVES, S. C. L.; VIEIRA-DA-SILVA, L. M. Atenção à saúde bucal e a descentralização da saúde no Brasil: estudo de dois casos exemplares no Estado da Bahia. Cadernos de Saúde Pública, Rio de Janeiro, v. 23, n. 5, p. 1119-1131, 2007.

CHAVES, S. C. L.; SILVA, L. M. V. As práticas profissionais no campo público de atenção à saúde bucal: o caso de dois municípios da Bahia. Ciência e Saúde Coletiva, Rio de Janeiro, v. 12, n. 6, p. 1697$1710,2007$.
PADILHA, W. W. N. et al. Planejamento e programação odontológico no Programa Saúde da Família do Estado da Paraíba: estudo qualitativo. Pesquisa Brasileira em Odontopediatria e Clínica Integrada, João Pessoa, v. 5, n. 1, p. 65-74, 2005.

SCATENA, J. H. G.; TANAKA, O. Y. Utilização do Sistema de Informações Hospitalares (SIH-SUS) e do Sistema de Informações Ambulatoriais (SIASUS) na análise da descentralização da saúde em Mato Grosso. Informe Epidemiológico do SUS, Brasília, DF, v. 10, n. 1, p. 19-30, 2001.

\section{SECRETARIA MUNICIPAL DE SAÚDE DE} FORTALEZA. A expansão do PSF em Fortaleza. Fortaleza, 21 de março de 2007. Disponível em: <http://www.sms.fortaleza.ce.gov.br/sms_v2/ Noticias_Detalhes.asp?noticia $=280>$. Acesso em: 29 abr. 2007.

SOUZA, T. M. S.; RONCALLI, A. G. Saúde bucal no Programa Saúde da Família: uma avaliação do modelo assistencial. Cadernos de Saúde Pública, Rio de Janeiro, v. 23, n. 11, p. 2727-2739, 2007. 